\title{
The impact of donor-recipient sex matching on transplant- related complications in children after allogeneic haematopoietic stem cell transplantation - a single-centre, retrospective study
}

\author{
Paweł Kutnik', Agnieszka Kwiatkowska', Dominika Krawczyk', Oliwia Polak', \\ Patryk Jawoszek', Daniel Puchała', Agnieszka Zaucha-Prażmo², Jerzy Kowalczyk² \\ 'Student Scientific Association at the Chair and Department of Paediatric Oncology, Haematology, and Transplantology, \\ Medical University of Lublin, Poland \\ 2Department of Paediatric Oncology, Haematology, and Transplantology, Medical University of Lublin, Poland
}

\section{ABSTRACT}

Introduction: Allogeneic haematopoietic stem cell transplantation (HSCT) is a well-established therapeutic option used in the treatment of malignant and non-malignant disorders. According to numerous studies, there is a correlation between donor sex or sex mismatching between donor and recipient and increased risk of graft-versus-host diseases.

Aim of the study: The aim of this study was to assess the impact of donor-recipient sex mismatching on the incidence of transplant-related complications in children undergoing allogeneic HSCT.

Material and methods: We conducted a retrospective study comparing the prevalence of transplant-related adverse events between female donor - female recipient, female donor - male recipient, male donor - male recipient, and male donor - female recipient groups. The study population included 198 patients from the department's database, who underwent allogeneic HSCT between 2001 and 2018 in the Department of Paediatric Haematology, Oncology, and Transplantology in Lublin.

Results: No statistically significant differences in prevalence of transplant-related complications between the four groups were found in this study: acute graft-versus-host disease $(p=0.53)$, chronic graft-versus-host disease $(p=0.69)$, hepatic veno-occlusive disease $(p=0.41)$, renal failure $(p=0.81)$, bleeding $(p=0.51)$, cytomegaly virus infection $(p=0.41)$, Epstein-Barr infection $(p=0.29)$, and fungal infection $(p=0.31)$.

Conclusions: The study showed no correlation between sex mismatching and HSCT complication frequency in children. The results of this study suggest that the donor's gender might not be a crucial factor in screening for potential donors in the paediatric population. Rising evidence of the lack of a direct impact of a donor's gender on overall HSCT transplant outcomes and adverse events in the paediatric population might in future change the criteria we follow when looking for potential donors. In conclusion, there is a need for prospective observational studies, which could investigate the mechanisms of adverse events and multicentre retrospective studies, which would allow larger homogeneous populations to be gathered to further research the impact of donor's demographics on allogeneic HSCT outcomes and complications.

\section{KEY WORDS:}

allo-HSCT, sex mismatching, donor gender.

\section{ADDRESS FOR CORRESPONDENCE:}

Paweł Kutnik, Student Scientific Association at the Chair and Department of Paediatric Oncology, Haematology, and Transplantology, Medical University of Lublin, 6 Gębali St., 20-093 Lublin, Poland, e-mail: pe.kutnik@gmail.com 


\section{INTRODUCTION}

Allogeneic haematopoietic stem cell transplantation (HSCT) is a well-established therapeutic option used in the treatment of many malignant and non-malignant disorders [1]. Matched sibling donors (MSD) are considered ideal donors, while matched unrelated donors (MUD) and mismatched family donors (MMFD) are one of the risk factors increasing the incidence of transplant-related complications. However, there are other factors that can increase the risk of post-transplant adverse events. According to numerous studies, there is a correlation between donor sex and increased risk of acute graftversus-host diseases (aGvHD) or sex mismatching between donor and recipient and increased occurrence of chronic graft-versus-host disease (cGvHD) $[2,3]$. The majority of those studies were conducted in adult populations, thus the impact of donor sex remains elusive in the paediatric population.

The latest research in the field of transplantation has focused on minor histocompatibility antigens (MiHA), which were reported to drive allo-immune response in graft-versus-host disease (GvHD) [4]. H-Y antigen, which belongs to the MiHA family, encoded on the Y chromosome, was associated with greater risk of GvHD in male recipients with female donor [5], which would explain the previously observed impact of sex mismatching between donor and recipient on transplant success. Contrarily, some studies reported no connection between donor sex and a higher prevalence of adverse events [6].

The aim of this study was to assess the impact of donor-recipient sex mismatching on the incidence of transplant-related complications in children undergoing allogeneic HSCT.

\section{MATERIAL AND METHODS}

This was a retrospective study approved by the Ethical Committee of the Medical University of Lublin (KE0254/15/2019). Given the nature of the study, the requirement for obtaining informed consent from each patient was waived.
The study population included 198 patients from the department database, who underwent allogeneic HSCT between 2001 and 2018 in the Department of Paediatric Haematology, Oncology, and Transplantology in Lublin, Poland. Reasons for needing allogenic transplant included: acute lymphoblastic leukaemia - 34.4\%; acute myeloid leukaemia - $18.3 \%$; severe aplastic anaemia - $18.3 \%$; severe combined immunodeficiency syndrome $-8.9 \%$; myelodysplastic syndrome - 7.8\%; non-Hodgkin lymphoma - 3.9\%; inborn errors of metabolism - 3.9\%; and primary immunodeficiency disease $-3.9 \%$.

The prophylaxis, diagnosis, and management of GvHD was provided according to the EBMT guidelines and centre procedures. The MSD patients received cyclosporin alone or in combination with methotrexate. The MUD patients received anti-thymocyte globulin as T-cell depletion. MMFD patients received ex-vivo T-cell depleted grafts.

\section{DESIGN FOR THE STUDY}

Patients treated with HSCT were divided into four groups according to the gender of the donor: female donor - female recipient (F-F), female donor - male recipient (F-M), male donor - female recipient (M-F), and male donor - male recipient (M-M) groups. Patients characteristics are presented in Table 1 . The median follow-up was 12 months.

The endpoints of the study were the occurrence rates of complications and included: acute graft-versus-host disease, chronic graft-versus-host disease, hepatic venoocclusive disease (VOD), renal failure, bleedings, cytomegaly virus infection (CMV), Epstein-Barr infection (EBV), and fungal infection.

\section{STATISTICAL ANALYSIS}

Statistic data was collected in Microsoft Excel spreadsheets (Microsoft, Redmond, WA, USA). Descriptive statistics are presented as median, minimums, and maximums for continuous variables and number with frequencies for categorical variables. Categorical variables

TABLE 1. Study group demographics

\begin{tabular}{|l|c|c|c|c|}
\hline Demographics & F-F $(n=38)$ & $\mathrm{F}-\mathrm{M}(n=43)$ & $\mathrm{M}-\mathrm{F}(n=53)$ & $\begin{array}{c}\mathrm{M}-\mathrm{M}(n=64) \\
n(\%)\end{array}$ \\
\hline Age $^{*}$ & $n(\%)$ & $n(\%)$ & $9(1-18)$ & $9(0-18)$ \\
PB & $9(0-18)$ & $8(0-17)$ & $17(32.1)$ & $15(23.4)$ \\
BM & $12(31.6)$ & $7(16.3)$ & $36(67.9)$ & $49(76.6)$ \\
MSD & $26(68.4)$ & $36(83.7)$ & $18(34)$ & $20(31.3)$ \\
MUD & $14(36.8)$ & $22(51.2)$ & $30(56.6)$ & $35(54.6)$ \\
MMFD & $21(55.3)$ & $18(41.9)$ & $5(9.4)$ & $9(14.1)$ \\
ABO mismatch & $3(7.9)$ & $3(6.9)$ & $25(47.2)$ & $30(46.9)$ \\
\hline
\end{tabular}

$F$ - $F$-female donor - female recipient, $F-M$ - female donor - male recipient, $M-F$ - male donor - female recipient, $M-M-$ male donor - male recipient, ${ }^{*}$ age is presented as the median value with minimum and maximum values in brackets, Other values presented as the number of patients in each group and the percentage of the entire group in brackets. Sources of stem cells: $P B-$ peripheral blood, $B M-$ bone marrow; ABO mismatch included: minor, major, and bi-directional mismatch. MSD - matched sibling donor, MUD - matched unrelated donor, MMFD - mismatched family donor 


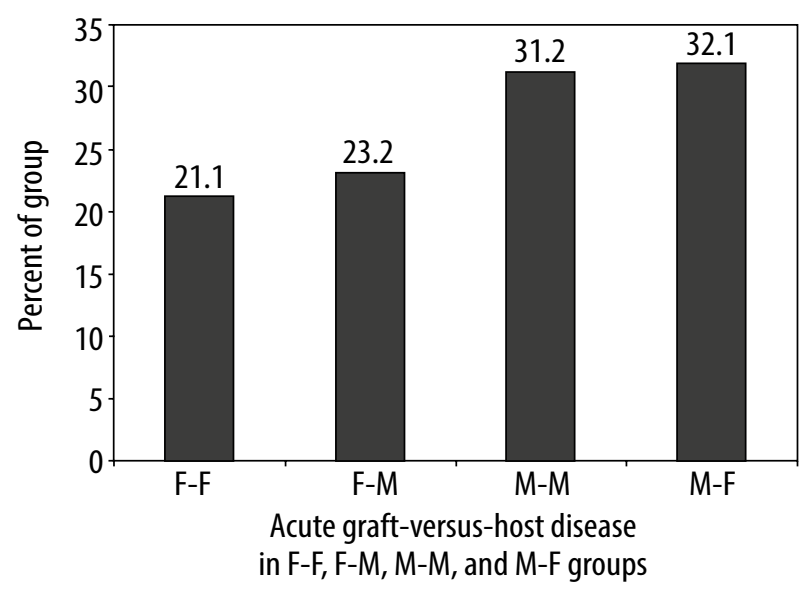

FIGURE 1. Occurrence of acute graft-versus-host disease in each group

are presented as the number of patients in which the complication occurred and frequencies in percentages (in brackets) and were analysed using the $\chi^{2}$ test. All statistical calculations were performed using Statistica 13.3 (StatSoft Inc., Tulsa, OK, USA).

\section{RESULTS}

Figure 1 presents absolute risks of aGvHD. The $X$-axis shows group populations: F-F, F-M, M-M, and M-F groups. Absolute risks are presented as frequencies in each group on the $Y$-axis. There were no statistically significant differences in occurrence of aGvHD across the groups. The number of patients who developed aGvHD were as follows: F-F 8 (21.1\%), F-M 10 (23.2\%), M-M 21 (31.2\%), and M-F 17 (32.1\%), $p$-value $=0.53$.

Figure 2 presents absolute risks of $\mathrm{cGvHD}$. The $X$-axis shows group populations: F-F, F-M, M-M, and M-F groups. Absolute risks are presented as frequencies in each group on the $Y$-axis. The cGvHD occurred in: four (10.5\%) patients in the F-F group, three (6.9\%) patients in the F-M group, nine (14.1\%) patients in the M-M group, and seven $(13.2 \%)$ patients in the M-F group, $p$-value $=0.69$.

Table 2 presents the occurrence, number of listed complications, and frequency in brackets in the F-F, F-M, $\mathrm{M}-\mathrm{M}$, and M-F groups. Values are number of patients in

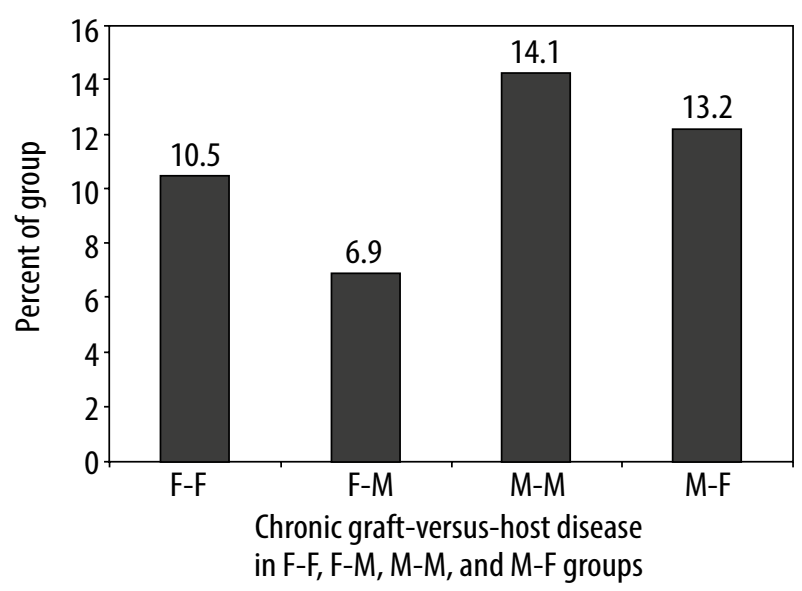

FIGURE 2. Occurrence of chronic graft-versus-host disease in each group

which the complication occurred and frequencies in percentages (in brackets). $p$-values were calculated using $\chi^{2}$ analysis. No statistically significant differences were observed across all four groups in the prevalence of infections, VOD, renal failure, or frequency of bleedings.

\section{DISCUSSION}

The presented study showed no correlation between donors' and recipients' genders and frequency of HSCT complications in children. There were no statistically significant differences in the prevalence of adverse events between all four groups, which corresponds with the findings of the latest study of 244 paediatric patients undergoing MSD HSCT, presented by Friedrich et al. in 2018 [7]. Rising evidence of lack of direct impact of sex-mismatching on overall HSCT transplant complications and outcomes in the paediatric population might in the future change the criteria we follow when looking for potential donors.

Due to the reports suggesting that female donor and male recipient mismatching is an independent risk factor of development of aGvHD and cGvHD, the comparison of prevalence of aGvHD and cGvHD are presented separately in charts 1 and 2. The higher occurrence rate of GvHD in female donor - male recipient sex mismatch, which was reported several times [8-10], was not ob-

TABLE 2. Infections, hepatic veno-occlusive disease, renal failure, bleeding

\begin{tabular}{|l|c|c|c|c|c|}
\hline Complication & $\begin{array}{c}\mathrm{F}-\mathrm{F}(n=38) \\
n(\%)\end{array}$ & $\begin{array}{c}\mathrm{F}-\mathrm{M}(n=43) \\
n(\%)\end{array}$ & $\begin{array}{c}\mathrm{M}-\mathrm{F}(n=53) \\
n(\%)\end{array}$ & $\begin{array}{c}\mathrm{M}-\mathrm{M}(n=64) \\
n(\%)\end{array}$ & $p$-value \\
\hline EBV infections & $4(10.5)$ & $2(4.7)$ & $9(17)$ & $9(14.1)$ & 0.29 \\
\hline CMV infections & $11(28.9)$ & $6(14)$ & $10(18.9)$ & $13(20.3)$ & 0.41 \\
\hline Fungal infections & $9(23.7)$ & $2(4.7)$ & $5(9.4)$ & $12(18.8)$ & 0.31 \\
\hline VOD & $7(18.4)$ & $4(9.3)$ & $4(7.5)$ & $7(10.9)$ & 0.41 \\
\hline Renal failure & $7(18.4)$ & $5(11.6)$ & $8(15.1)$ & $8(12.5)$ & 0.81 \\
\hline Bleedings & $2(5.2)$ & $2(4.7)$ & $5(9.4)$ & $2(3.1)$ & 0.51 \\
\hline
\end{tabular}

$F-F$ - female donor - female recipient, $F-M$ - female donor - male recipient, $M-F$ - male donor - female recipient, $M-M$ - male donor - male recipient, EBV - Epstein-Barr virus, CMV - cytomegaly virus, $V O D$ - hepatic veno-occlusive disease, $p$-values were calculated using $X^{2}$ analysis 
served. It might advocate that not gender, but potential autoimmunisation related to gender, for example, parous status of female donors, is the main factor in creating adverse events, which was suggested in the past [11-13] and needs further investigations. Additionally, in our study none of the groups showed any statistically significant differences in occurrence of any researched complication.

The results of this study suggest that the donor's gender might not be a crucial factor in screening for potential donors in the paediatric population. The donor's gender could have an impact on transplant outcome and occurrence rate of adverse events in certain types of diseases or levels of matching; however, definitive answers require a study with a more homogenous population, which might not be feasible in single-centre studies.

This study has numerous limitations. The retrospective nature of this study did not allow us to further investigate the mechanism of adverse events, including testing for MiHA in patients developing GvHD. We did not have the full demographic characteristics of all donors; therefore, the age, parous status, and previous medical history were not taken into account in the analysis. The study population was not homogeneous. Patients received transplants from related and unrelated donors. Primary diseases varied in study groups. The extended time of collected data between 2001 and 2018, which allowed the presentation of a larger study population, could have affected the results due to the change of guidelines and standard of care during that period. The source of stem cells was also not taken into account due to a relatively small group of peripheral blood patients, and peripheral blood transplant is considered an independent factor of development of cGvHD [14]; therefore, we cannot exclude the potential impact of the source of stem cells on our results.

The data presented in this study provide valuable insight on the paediatric HSCT population, which might differ from the adult HSCT population. Of note, the research on this topic revealed a lack of definitive findings on the direct impact of the donor's gender on transplant complication rates and outcome, especially in the paediatric population, where evidence in even more insufficient.

\section{CONCLUSIONS}

In conclusion, there is a need for further research on the impact of the donor's demographics on HSCT outcomes and adverse events, especially prospective observational studies, which would allow investigation of mechanisms of transplant-related complications, and multicentre retrospective studies that would support gathering larger and more homogeneous populations, as performed for multiple myeloma [15].

\section{REFERENCES}

1. Styczyński J, Balwierz W, Dembowska-Bagińska B, et al. Paediatric oncology and haematology in Poland: position paper. Pediatr Pol 2018; 93: 451-461.

2. Nannya Y, Kataoka K, Hangaishi A, et al. The negative impact of female donor/male recipient combination in allogeneic hematopoietic stem cell transplantation depends on disease risk. Transpl Int 2011; 24: 469-476.

3. Randolph SS, Gooley TA, Warren EH, et al. Female donors contribute to a selective graft-versus-leukemia effect in male recipients of HLA-matched, related hematopoietic stem cell transplants. Blood 2004; 103: 347-352.

4. Goulmy E, Schipper R, Pool J, et al. Mismatches of minor histocompatibility antigens between HLA-identical donors and recipients and the development of graft-versus-host disease after bone marrow transplantation. N Engl J Med 1996; 334: 281-285.

5. Markiewicz M, Siekiera U, Dzierzak-Mietla M, et al. The impact of $\mathrm{H}-\mathrm{Y}$ mismatches on results of HLA-matched unrelated allogeneic hematopoietic stem cell transplantation. Transplant Proc 2010; 42: 3297-3300.

6. Przepiorka D, Smith TL, Folloder J, et al. Risk factors for acute graft-versus-host disease after allogeneic blood stem cell transplantation. Blood 1999; 94: 1465-1470.

7. Friedrich P, Guerra-Garcia P, Stetson A, et al. Young Female Donors Do Not Increase the Risk of Graft-versus-Host Disease or Impact Overall Outcomes in Pediatric HLA-Matched Sibling Hematopoietic Stem Cell Transplantation. Biol Blood Marrow Transplant 2018; 24: 96-102.

8. Verdijk RM, Kloosterman A, Pool J, et al. Pregnancy induces minor histocompatibility antigen-specific cytotoxic T cells: implications for stem cell transplantation and immunotherapy. Blood 2004; 103 : 1961-1964.

9. Linscheid C, Petroff MG. Minor histocompatibility antigens and the maternal immune response to the fetus during pregnancy. Am J Reprod Immunol 2013; 69: 304-314.

10. van Kampen CA, Versteeg-van der Voort MF, Langerak-Langerak J, et al. Pregnancy can induce long-persisting primed CTLs specific for inherited paternal HLA antigens. Hum Immunol 2001; 62: 201207.

11. Remberger M, Kumlien G, Aschan J, et al. Risk factors for moderate-to-severe chronic graft-versus-host disease after allogeneic hematopoietic stem cell transplantation. Biol Blood Marrow Transplant 2002; 8: 674-682.

12. Bross DS, Tutschka PJ, Farmer ER, et al. Predictive factors for acute graft-versus-host disease in patients transplanted with HLA-identical bone marrow. Blood 1984; 63: 1265-1270.

13. Randolph SSB, Gooley TA, Warren EH, et al. Female donors contribute to a selective graft-versusleukemia effect in male recipients of HLA-matched, related hematopoietic stem cell transplants. Blood 2004; 103: 347-352.

14. Anasetti C, Logan BR, Lee SJ, et al. Peripheral-Blood Stem Cells versus Bone Marrow from Unrelated Donors. N Eng J Med 2012; 367: 1487-1496.

15. Gahrton G, Iacobelli S, Apperley J, et al. The impact of donor gender on outcome of allogeneic hematopoietic stem cell transplantation for multiple myeloma: reduced relapse risk in female to male transplants. Bone Marrow Transplant 2005; 35: 609-617.

\section{DISCLOSURE}

The authors declare no conflict of interest. 